\title{
Delays in the submission of new drugs in Canada
}

\author{
Ali Shajarizadeh MA, Aidan Hollis PhD
}

$\mathrm{S}$ ome commentators have claimed that Health Canada's process for approving new drugs is excessively slow, thereby delaying access to these drugs by Canadians. ${ }^{1}$ However, the submission of new drugs to Health Canada for approval is systematically delayed compared with submissions to regulatory agencies in the United States and the European Union, which delays the availability of new drugs in Canada. In this paper, we analyze the timing of approvals and submissions in Canada and explore possible reasons for delays based on available data. We also explore the likely effects of a harmonized process for submissions between the US Food and Drug Administration (FDA) and Health Canada.

\section{What is behind the apparent delays in the approval of new drugs in Canada?}

We began our analysis by searching the drug databases of the FDA, the European Medicines Agency and Health Canada to obtain information about drugs with new molecular entities or new active substances that were approved by at least one of these agencies between 2000 and 2011. Missing submission dates for drugs approved in Canada were provided by Health Canada. (Details of our methods are provided in Appendix 1, available at www.cmaj.ca/lookup/suppl /doi:10.1503/cmaj.130814/-/DC1.) The initial sample of 380 drugs included a subset of 111 drugs submitted to the FDA or the European Medicines Agency that were not submitted for approval to Health Canada (Figure 1). Hence, the approval of the 111 drugs in Canada would be infinitely delayed. Because we focused our analysis on the reasons for delays in Canada, we analyzed data only for the 259 drugs that were submitted to Health Canada and to at least one of the other two agencies. We compared the submission delay (the interval between the first submission of a drug to any of the three regulatory agencies and the submission date in a specific jurisdiction) and the time to approval (the interval between the submission date and the date of authorization to launch for market) for new drugs in each jurisdiction using the data provided in Appendix 1.

We found that differences across jurisdictions in approval-processing times played a small role in the delays. However, differences in the timing of drug submissions were an important factor (Table 1). Although the mean time to approval was about 90 days longer in Canada than in the US or the EU, the mean submission delay in Canada was much longer than in the other two jurisdictions. The mean submission delay hides considerable variation: many drugs were submitted to Health Canada with no substantial delay, but others were delayed by more than two years. New drugs reached the market much later in Canada than in the US and the EU because of long delays before their submission to Health Canada. Figure 2 shows the distribution of submission delays in the three jurisdictions. In the US and EU, most new drugs were submitted within three months after their first submission to any of the three jurisdictions. In Canada, about $70 \%$ of the new drugs were submitted more than three months, and $40 \%$ more than one year, after their first submission. For drugs that were ultimately approved in Canada and in at least one of the other jurisdictions, the mean delay from first submission in either foreign jurisdiction to submission in Canada was 540 days.

\section{KEY POINTS}

- Accessibility to new drugs in Canada is delayed primarily because of delays in submission to Health Canada by pharmaceutical companies and not because of a longer approval-processing time at Health Canada.

- For drugs approved in Canada and in the European Union or the United States or both, the mean delay from first submission in the foreign jurisdiction until submission in Canada was 540 days; on average, the processing of approvals by Health Canada was 90 days longer than the approval-processing time at the FDA or the European Medicines Agency.

- Corporate capacity of the pharmaceutical companies and priority status of new drugs appear to be the most important determinants of submission delays.

- Harmonization of the regulatory processes of the FDA and Health Canada may accelerate new drug submissions in Canada. 


\section{What are the reasons for delays in submissions to Health Canada?}

We propose four hypotheses that may explain the delays in the submission of new drugs to Health Canada. First, the data requirements for submissions may be more onerous in Canada, causing pharmaceutical companies to delay submisions. It may take longer to assemble a successful application for Health Canada than to assemble one for the FDA or the European Medicines Agency. We call this the "stringency" explanation. Second, companies may delay their submissions to Health Canada because the value of getting listed quickly in Canada is small relative to the potential harm to the regulatory process in the other jurisdictions in the event that Health Canada seeks additional information. We call this the "risk" hypothesis. Third, companies may have limited capacity to make submissions and therefore prioritize submissions by market according to profitability. Therefore, larger markets would attract the first submissions. We call this the "capacity" explanation. Fourth, companies may

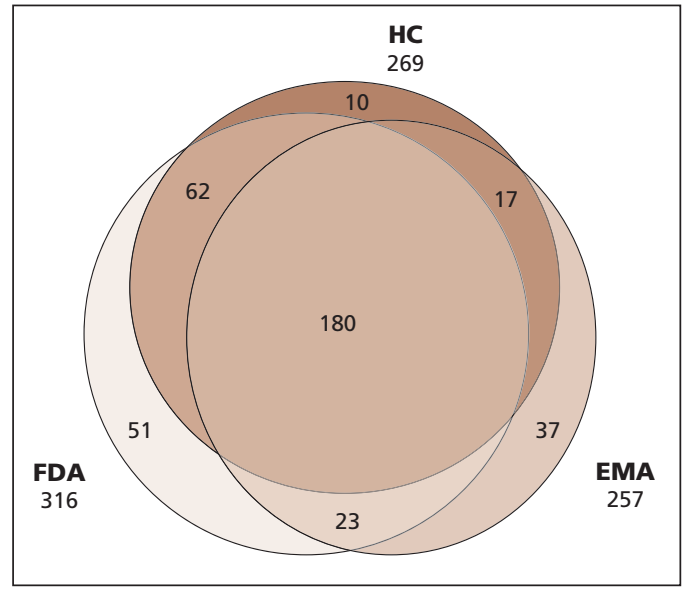

Figure 1: Number of drug approvals by Health Canada (HC), the US Food and Drug Administration (FDA) and the European Medicines Agency (EMA) between 2000 and 2011. intentionally delay their submissions to Health Canada because they want to obtain approval for their_drugs in high-priced markets first to obtain an attractive price that other countries will use as a reference. We call this the "price" explanation.

\section{Stringency}

We explored the stringency hypothesis by first contacting officials at Health Canada. They stated that the standards of what constitutes an adequate submission may differ for individual drugs but, on average, are roughly the same across jurisdictions for standard drugs.

The FDA offers programs that may enable early submission for new drugs for serious conditions where no acceptable treatment exists: fasttrack status and accelerated approval. Fast-track status offers a rolling review in which modules of the application are submitted as they are completed. For these cases, the date of submission of the first module is considered the date of submission for the application. Accelerated approval allows firms to use surrogate outcomes that are not well-established. ${ }^{2}$ These programs allow companies to submit what are essentially incomplete applications, which would generally not be acceptable at Health Canada. (Health Canada is currently developing a program for orphan drugs. Consideration will be given to the small size of the patient population for the development, evaluation and approval of these drugs. ${ }^{3}$ ) These FDA programs are heavily used for orphan drugs, as reported by Seoane-Vazquez and colleagues. ${ }^{4}$ In 2004, the European Medicines Agency created a similar program (conditional marketing authorizations) that permits submissions for new drugs that address unmet medical needs, even when comprehensive clinical data have not been provided. However, conditional authorizations were not empirically important during the period of our analysis: as Joppi and colleagues ${ }^{5}$ showed, only 2 of the 44 orphan medical products approved by the European Medicines Agency between 2000 and 2007 had conditional authorizations.

Table 1: Submission delays and times to approval for new drugs submitted for approval in Canada and at least one other jurisdiction

\begin{tabular}{|c|c|c|c|c|c|c|c|}
\hline \multirow[b]{2}{*}{ Jurisdiction } & \multirow{2}{*}{$\begin{array}{l}\text { No. of } \\
\text { drugs }\end{array}$} & \multicolumn{3}{|c|}{ Submission delay, ${ }^{*} d$} & \multicolumn{3}{|c|}{ Time to approval, $+\mathrm{d}$} \\
\hline & & Mean & Median & SD & Mean & Median & SD \\
\hline Canada & 259 & 540 & 180 & 810 & 551 & 442 & 344 \\
\hline United States & 242 & 106 & 0 & 462 & 461 & 308 & 339 \\
\hline European Union & 197 & 215 & 12 & 561 & 464 & 451 & 136 \\
\hline
\end{tabular}

Note: $\mathrm{SD}=$ standard deviation.

*The interval between the date of first submission to a regulatory agency in any of the three jurisdictions and the date of submission in a specific jurisdiction.

tThe interval between the submission date and the date of authorization to launch for market in each jurisdiction. 
To determine the extent to which the stringency hypothesis explained delays in submission to Health Canada, we analyzed our data according to whether the drugs had an orphan status in the US. We anticipated that drugs with an orphan classification in the US would have a relatively short submission delay in the US compared with the submission delays in Canada and the EU, which we expected would be similar. In general, orphan drugs were submitted much later in Canada than in the EU and the US (Figure 3). Many orphan drugs are never submitted to Health Canada. For those that are, it would seem advantageous to the company to submit as early as possible. We found little difference in the timing of submissions between orphan and nonorphan drugs within each jurisdiction, and a large, consistent difference between jurisdictions regardless of orphan status. We conclude that, although stringency may have had some impact on the timing of submissions, it was not a strong factor for differences in submission delays between jurisdictions.

\section{Risk}

The risk that Health Canada might request additional information or the results of new studies and that such requests might have a "contagion" effect on other regulatory authorities and cause delays in the approval process in those jurisdictions could lead pharmaceutical companies to delay submission in Canada until they have gained approval for the drug in other jurisdictions. To investigate this hypothesis, we considered whether the timing of submissions in Canada was associated with marketing approval of the drugs in the US and the EU.

Of the 259 new drugs submitted to Health Canada in our sample, 88 had previously been approved in the US and 45 had been approved in the EU. Figure 4 shows the distribution of the time difference between approval in the US or EU and submission to Health Canada. Most of the drugs were submitted to Health Canada before they were approved in the other jurisdictions (i.e., a negative time difference). If the risk hypothesis were correct, we would expect to see a jump in the number of submissions to Health Canada at the time of approval in the other jurisdictions (time zero). Because no noticeable increase was observed (Figure 4), we conclude that the risk hypothesis does not explain submission delays in Canada.

\section{Capacity}

Bringing a new drug to market is costly and requires considerable expertise in the regulatory process. Health Canada's fee for submission of a new drug is $\$ 322056 .{ }^{6}$ Not all companies have the capacity inside Canada to navigate the regu- latory submission process. Larger companies are more likely than smaller ones to have dedicated staff in Canada with such expertise. Therefore, smaller companies may choose to prioritize their submissions to larger markets.

To explore the capacity hypothesis, we compared submission delays by size of pharmaceutical company. Larger companies were those ranked as the top 30 in terms of sales (ranked by SCRIP Intelligence, www.scrip100.com). Half of the 259 new drugs in our sample were marketed by these companies.

Differences in submission delays were evident between the larger and smaller companies (Figure 5). Applications from larger companies were $20 \%$ more likely than those from smaller companies to be submitted to Health Canada within six months after the first submission to the FDA or the European Medicines Agency. In addition, the probability of a submission delay longer than two years was $15 \%$ lower among larger compa-

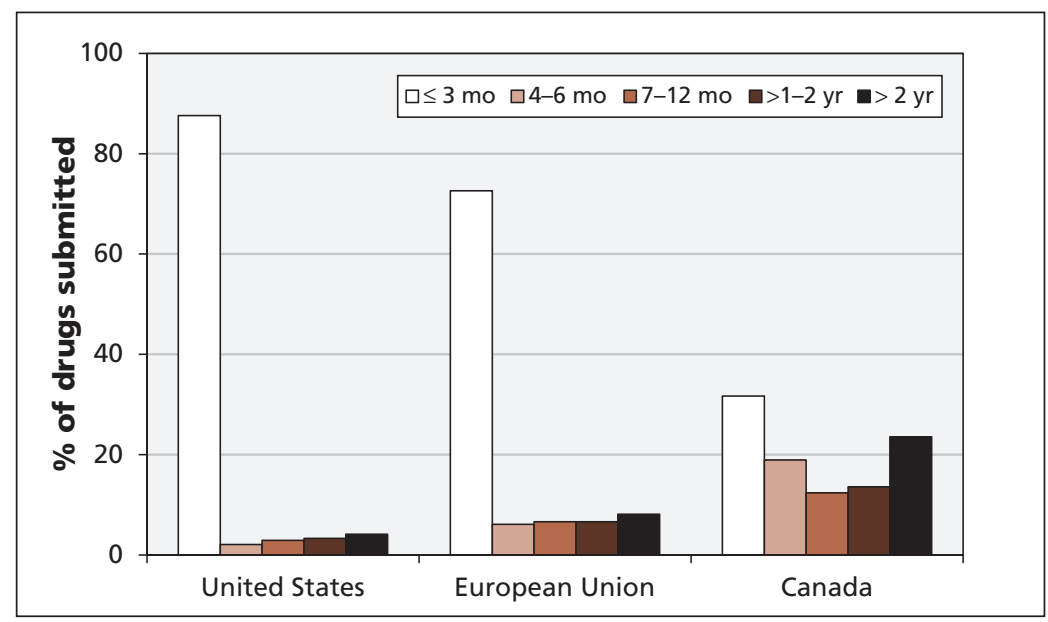

Figure 2: Delays in the submission of new drugs in the United States, the European Union and Canada. A submission delay is the interval between the date a drug is first submitted to any of the three jurisdictions and the submission date in a specific jurisdiction.

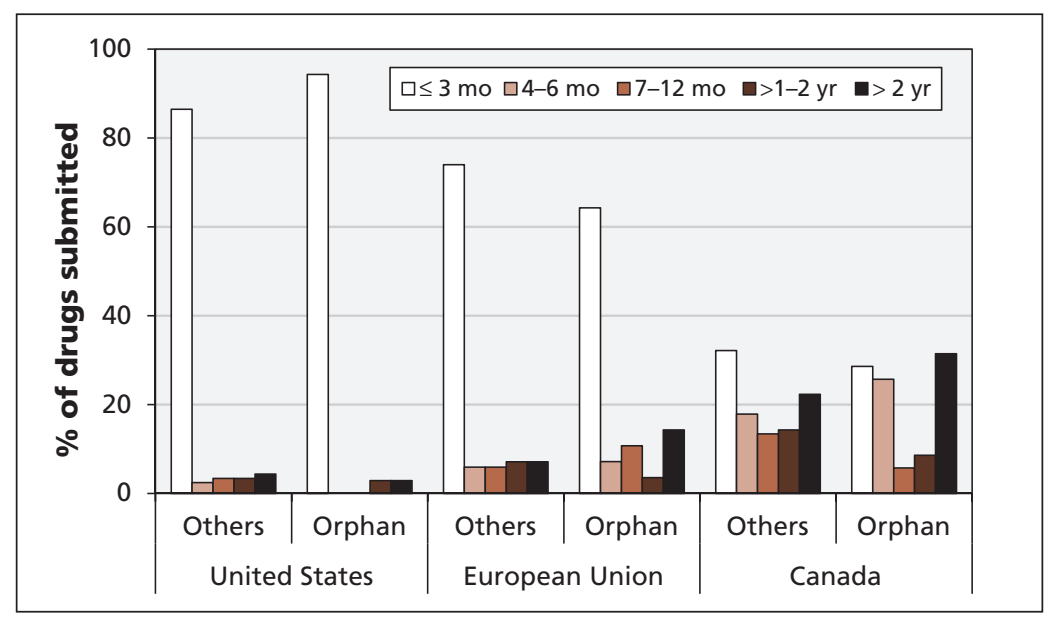

Figure 3: Delays in the submission of new drugs by orphan status. 
nies. Although smaller companies had longer submission delays than larger companies had, this was not the entire story: submission delays were

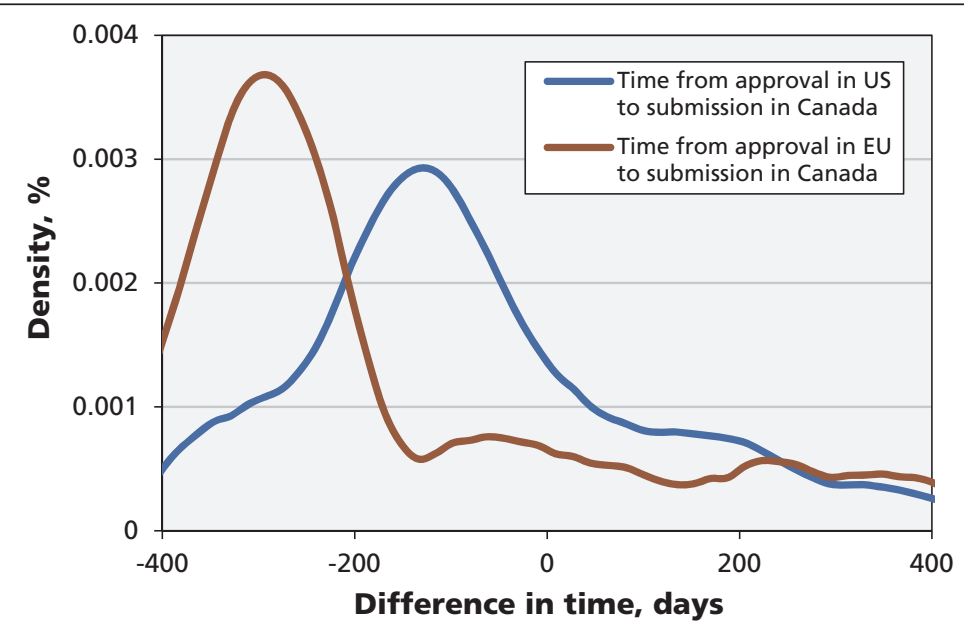

Figure 4: Distribution of the time difference between drug approval in the United States or European Union and submission for approval in Canada.

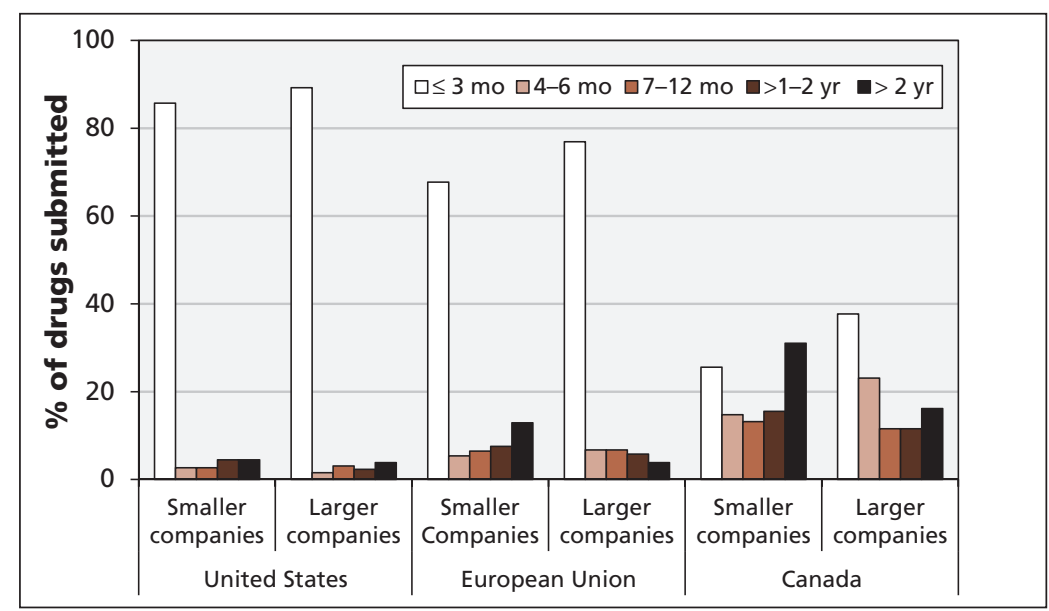

Figure 5: Delays in the submission of new drugs by company size. Larger companies = top 30 companies in terms of sales (ranked by SCRIP Intelligence).

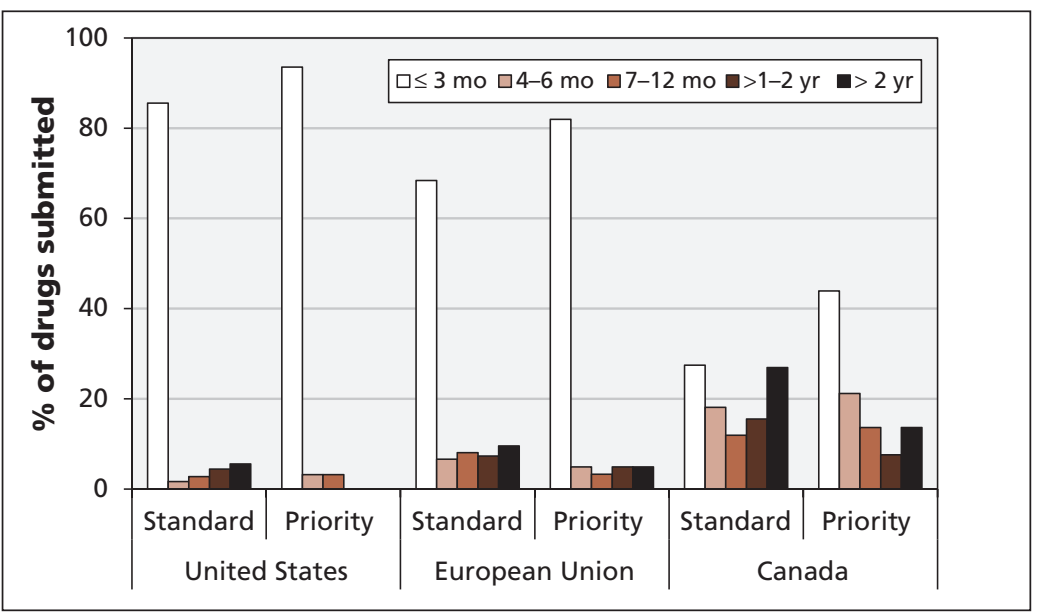

Figure 6: Delays in the submission of new drugs by review status. Priority = first-in-class drugs, standard = all other new drugs. greater in Canada than in the US and EU for both large and small companies, and the difference in submission delays between Canada and the other jurisdictions was much larger than the difference between large and small companies.

\section{Price}

Many countries consider prices in other markets when deciding what they are willing to pay for a new drug, particularly for the first drug in a class. The US is the ideal reference market if com panies do in fact delay their submissions to Health Canada until they get initial approval in this higher priced market. International price referencing is important for first-in-class drugs but not as important for subsequent class entrants (i.e., me-too or follow-on drugs), because prices within a class are typically determined by the pioneer in the class in most markets, and follow-on drugs are priced at about the same level. ${ }^{7}$ This distinction is formally built into the framework for price review of Canada's Patented Medicine Prices Review Board. ${ }^{8}$ Therefore, in our analysis of the price hypothesis, we expected first-in-class drugs to have longer submission delays than follow-on drugs in Canada.

Figure 6 shows the distribution of submission delays for new drugs according to their review status in Canada (priority status for first-in-class drugs and standard status for all other new drugs). Of the 259 new drugs submitted to Health Canada in our sample, 66 were recognized by the agency as having priority status. The submission delay was less than 3 months for $44 \%$ of these drugs, four to six months for $21 \%$ and seven months or longer for $36 \%$. The submission delays in Canada for standard drugs were $27 \%, 18 \%$ and $55 \%$, respectively. In effect, drugs with priority status typically were submitted to Health Canada with less of a delay than standard drugs were. Thus, we conclude that the price hypothesis does not explain the submission delays in Canada.

\section{Joint analysis}

We considered the combined effect of various factors by further examining our dataset using the econometrics model described in Appendix 1. For orphan drugs, our analysis showed that the size of the company had a substantial effect on the timing of a submission, with a mean delay of about 14 months for submissions to Health Canada from smaller companies. The delay was considerably shorter for nonorphan drugs and for submissions by large companies. Furthermore, drugs with priority review status were, on average, submitted earlier than follow-on drugs both in Canada (about one year earlier) and in other jurisdictions (seven 
months earlier), regardless of company size and seemingly without international price referencing as a contributing factor. The first choice for market submissions appears to be the US, with firms submitting their applications to the FDA 4 months earlier on average than their submissions to the European Medicines Agency and 14 months earlier on average than their submissions to Health Canada.

\section{Canada compared with other smaller markets}

In the previous sections, we compared the two largest markets in the world with Canada and found that corporate capacity appeared to be an important determinant of submission delays. If this is the case, other relatively small markets would be expected to experience delays similar to Canada's. Few countries could be used for comparison because submission dates are considered confidential in most jurisdictions. We chose Australia because its Therapeutic Goods Administration publishes years of submission for some new drugs (mostly those submitted after 2007) in their public assessment reports. ${ }^{9}$ We compared the years for drugs submitted to both Australia and Canada $(n=38)$ with the years of submission in the US and EU (Appendix 1). We found that new drugs were submitted for approval to Australia after they were submitted to Canada. Because the market is somewhat smaller in Australia than in Canada, this finding supports the corporate capacity hypothesis.

\section{What can be done to speed up drug submissions in Canada?}

Health Canada is currently working with the FDA to develop a harmonized system for new drug submissions. The harmonized system will enable companies to use the same electronic portal to submit applications to both Health Canada and the FDA, which may accelerate the approval process. ${ }^{10}$ This system will also incorporate a new pathway for the approval of orphan drugs in Canada. Although the new portal will facilitate submissions to both agencies, there is no requirement to submit to both at the same time. Therefore, if the reason for delaying a submission is a lack of global regulatory capacity, the approval process will be accelerated. Our analysis suggests that harmonized submission for the FDA and Health Canada will accelerate the arrival of new drugs in Canada, mainly for smaller companies that appear to delay because of lower capacity for submissions.

\section{Conclusion}

We examined several possible reasons for delays in the submission of new drug applications to Health Canada, and our findings are revealing. We found that corporate capacity and priority status of new drugs are important determinants of submission delay. We believe that the harmonization of the regulatory processes of the FDA and Health Canada may accelerate drug submissions in Canada. However, because the situation is different for every drug, there may be other reasons for delaying (or not delaying) a submission to Health Canada that have not been covered in this article. Each regulatory domain varies in terms of its health care system, insurance coverage and regulatory approach. We examined differences in submission delays between orphan and nonorphan drugs and between first-in-class and follow-on drugs, but other differences may also affect the process.

\section{References}

1. Rovere M, Skinner B. Access delayed, access denied 2012: waiting for new medicines in Canada. Vancouver (BC): Fraser Institute; 2012. Available: www.fraserinstitute.org/uploadedFiles /fraser-ca/Content/research-news/research/publications/access -delayed-access-denied-2012.pdf (accessed 2013 Nov. 15).

2. Field M, Boat T, editors; Committee on Accelerating Rare Diseases Research and Orphan Product Development, Institute of Medicine. Rare diseases and orphan products: accelerating research and development. Washington (DC): National Academies Press; 2010.

3. An orphan drug framework for Canada. Ottawa (ON): Health Canada; 2012. Available: www.hc-sc.gc.ca/ahc-asc/media/nr-cp / 2012/2012-147a-eng.php (accessed 2013 Nov. 11).

4. Seoane-Vazquez E, Rodriguez-Monguio R, Szeinbach S, et al Incentives for orphan drug research and development in the United States. Orphanet J Rare Dis 2008;3:33.

5. Joppi R, Bertele V, Garattini S. Orphan drug development is not taking off. Br J Clin Pharmacol 2009;67:494-502.

6. Pharmaceutical submission and application review. Ottawa $(\mathrm{ON})$ : Health Canada. Available: www.hc-sc.gc.ca/dhp-mps /finance/fees-frais/pharma-eng.php (accessed 2014 Apr. 22).

7. Dylst P, Vulto A, Simoens S. Reference pricing systems in Europe: characteristics and consequences. GaBI J 2012;1:127-31.

8. Compendium of policies, guidelines and procedures - reissued June 2013. Ottawa (ON): Patented Medicine Prices Review Board; 2013. Available: www.pmprb-cepmb.gc.ca/english /View.asp? $\mathrm{x}=1733$ (accessed 2013 Nov. 15).

9. Australian public assessment reports for prescription medicines. Canberra (Australia): Department of Health: Therapeutic Goods Administration; 2014. Available: www.tga.gov.au/industry/pm -auspar-active.htm (accessed 2013 Nov. 15).

10. RCC Personal Care Products and Pharmaceuticals Working Group. Common electronic submissions gateway (ESG) work plan. Ottawa (ON): Health Canada; 2012. Available: www .actionplan.gc.ca/grfx/BAP-RCC/CESG_Reformat_Eng.pdf (accessed 2013 May 21).

Affiliation: Department of Economics, University of Calgary, Calgary, Alta.

Contributors: Both authors contributed substantially to the conception and design of the study and the acquisition, analysis and interpretation of the data. Both drafted the article and critically revised it for important intellectual content-and approved the final version of the manuscript submitted for publication.

Acknowledgement: The authors were supported by a Canadian Institutes of Health Research Emerging Team Grant on Developing Effective Policies for Managing Technologies for Rare Diseases. 